\title{
Preparation of Eight Strands Hamstring Tendon Graft for Anterior Cruciate Ligament Reconstruction Regardless Fixation Methods: Technical Note
}

\author{
Emad Mureed Shohdy ${ }^{*}$ \\ Division of Sport Medicine, Department of Orthopaedic Surgery, Al Razi Orthopaedic Hospital, Kuwait
}

\begin{abstract}
Background: Anterior cruciate ligament reconstruction is one of the most commonly performed procedures in orthopedics. There are several reconstruction graft choices that can be used to reconstruct the anterior cruciate ligament, with autograft hamstring tendons being one of the most commonly used.
\end{abstract}

Study Design: Technical note.

Purpose: The purpose of this paper is to describe in details eight strands hamstring graft preparation technique to increase graft diameter for anterior cruciate ligament reconstruction.

Methods: Hamstring tendons one of the most commonly used graft for anterior cruciate ligament reconstruction. Preparation of a hamstring graft varies according to patient characteristics and surgeon preference. It is common for the tendons to be folded over each other in order to increase the thickness of the donor graft. In order for the folded tendons to act as one unit they are sutured together using a whip stitch technique.

Results: The option adopted in the present technique was to make quadrupled each one of hamstring tendons to get eight strands $A C L$ graft, which led to increase graft diameter size which gives higher knee stability and long-term survival of the graft.

Conclusion: Using this technique increasing graft diameter size by increasing number of folds regardless fixation methods, to ensure long-term survival of small-sized hamstring tendon graft tissue which is common in our population especially non-athletic.

\section{Keywords}

Anterior cruciate ligament, Hamstring autograft, Preparation technique, Graft diameter

\section{Introduction}

The anterior cruciate ligament $(A C L)$ is one of the most commonly injured structures of the knee [1]. Several different methods have been used for the treatment of the unstable anterior cruciate ligament deficient knee. These methods differ in the surgical techniques and in the type of graft used (autograft, allograft and synthetic) [2]. The hamstring tendon autograft has been used as an alternative to the BPTB graft due to the reduced donor-site morbidity and significantly improved fixation technique and are currently considered as one of the most common grafts used in ACL reconstruction [3].

Certain conditions such as multiplicity of ligaments injuries and small-sized diameter of the harvested grafts which are common in our population led to developing of this technique to obtaining higher knee stability and to ensure longterm survival of small-sized hamstring tendon graft tissue by increasing number of folds of tendon to obtain larger graft diameter. It is common for the tendons to be folded over each other in order to increase the thickness of the donor graft. In order for the folded tendons to act as one unit they are sutured together using a whip stitch technique [4]. The donor graft is then passed through the tibial tunnel and into the femoral tunnel and fixed using a variety of fixation meth-

*Corresponding author: Emad mureed shohdy, Division of Sport Medicine, Department of Orthopaedic Surgery, Al Razi Orthopaedic Hospital, Kuwait City, Kuwait

Accepted: September 08, 2020

Published online: September 10, 2020

Citation: Shohdy EM (2020) Preparation of Eight Strands Hamstring Tendon Graft for Anterior Cruciate Ligament Reconstruction Regardless Fixation Methods: Technical Note. J Orthop Surg Tech 3(2):140-142 
ods including screws, suspensory apparatus and transfixion devices which may be metallic, polymer or bio-absorbable. Commonly, both hamstring tendons are doubled to get quadrupled graft, even with this technique in case of small sized tendon, the graft diameter size maybe less than $8 \mathrm{~mm}$ which may led to early graft failure [5].

In this technique, each tendon is quadrupled to get 8 strands ACL graft with diameter more than $8 \mathrm{~mm}$ regardless fixation methods. In addition, quadrupled semitendinosus tendon can be used alone for $\mathrm{ACL}$ graft and gracilis tendon can be used for anterolateral ligament (ALL) reconstruction.

\section{Surgical Technique}

\section{Graft harvest}

For hamstring tendon autograft, the semitendinosus (ST) and/or gracilis (GC) can be harvested from the surgical side or the contralateral side. A vertical anteromedial incision at the level of the tibial tubercle is used to expose the sartorius fascia, which covers the hamstring tendons. An incision in the sartorial fascia permits the exposure of the tendons. The tendons are recognized and then placed separately through a tendon stripper and released from its muscular attachment proximally while bending the knee in $90^{\circ}$ and advancing the stripper in a proximal direction.

\section{Graft preparation}

The graft preparation and configuration is easily done in a graft preparation system Acufex graft master III (Smith \& Nephew, Andover, MA). Removal of excess muscular tissue from each tendon is done and unstable parts of the tendon are removed. If ST and GC tendons are harvested, they should be twisted in a reverse orientation in order that the proximal end of the ST is nearby to the distal end of the GC graft and vice versa then each end whip stitched. The graft(s) are estimated in a graft preparation system and measurements of length of each harvested tendon(s) are made to define which graft preparation method is more suitable for that specific $\mathrm{ACL}$ reconstruction. The graft configuration is then done (folded) to determine the appropriate graft length and diameter.

\section{Eight-strand graft configuration}

Each tendon can be whip stitched separately or whip stitched together in a reverse orientation (proximal end of the

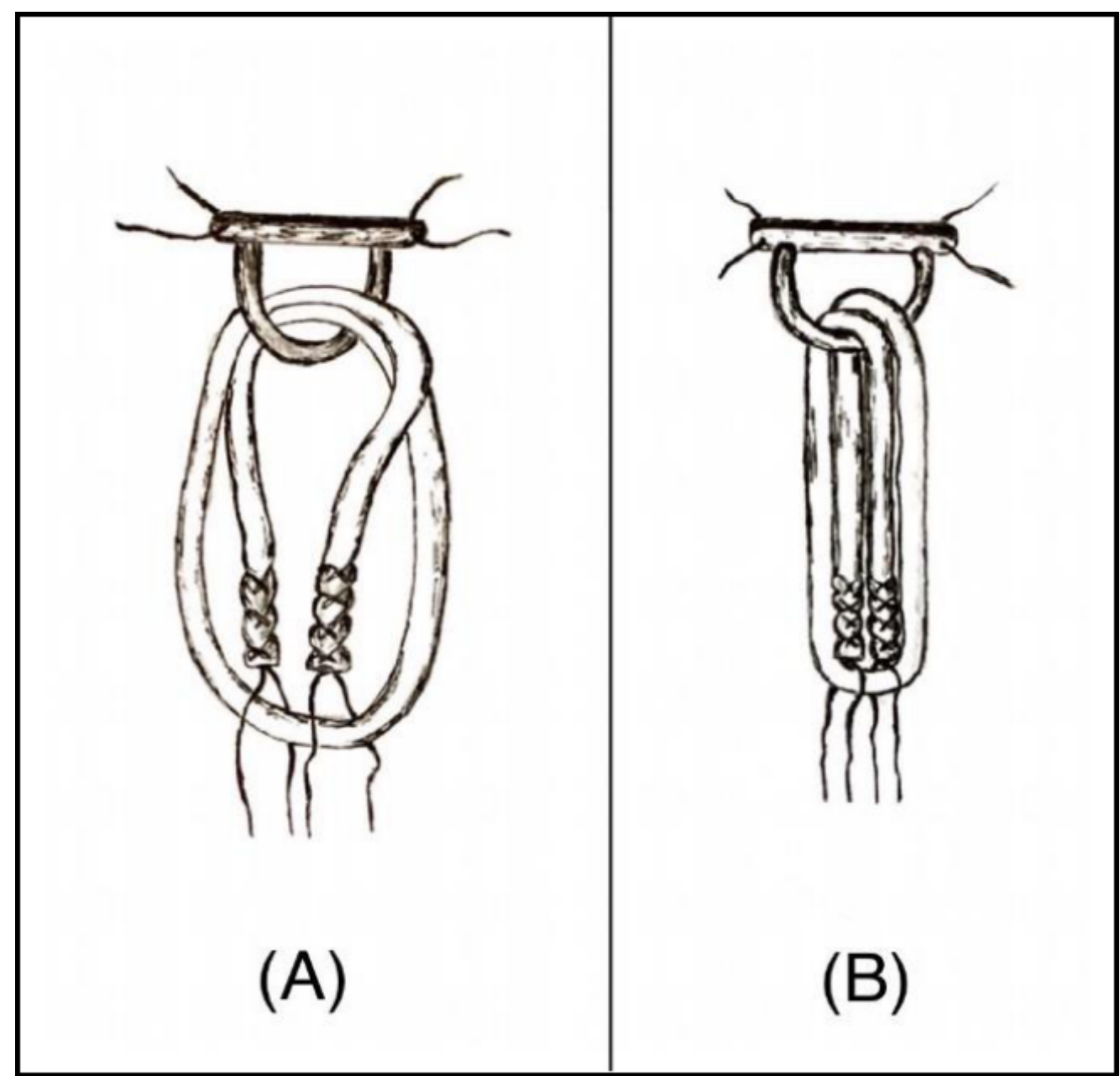

Figure 1: A drawing of quadrupled tendon graft (A) Each end of the tendon loaded in suspensory device to get 4 strands with one limb of whip stitch suture from each free end is passed through looped part of tendon; (B) Then 4 limbs of whip stitched suture are pulled to tensioning the graft. 
ST is adjacent to distal end of the GC), then each end of the tendon loaded in suspensory device or folded over Ethibond suture (if Biointrafix interference screw will be used for femoral fixation) to get 4 strand (Video 1 , which demonstrates hamstring graft preparation techniques, showing the ST and the GC whip stitched together with Ethibond suture augmented by Mersilene tape in a reverse orientation), (Video 2, Hamstring graft preparation technique, showing the ST and the GC whip stitched with Ethibond suture separately). One limb of whip stitch suture from each free end is passed through looped part of graft, and then 4 limbs of whip stitched suture are pulled to tensioning the graft (Figure 1). This technique can also be performed with 2 cortical suspensory adjustable length devices for an all-inside $A C L$ reconstruction, by tying whip stitch suture from each end of graft together.

\section{Discussion}

Hamstring tendons are one of the more commonly used grafts for $\mathrm{ACL}$ reconstruction, the semitendinosus tendon with or without the gracilis tendon is harvested [4]. The resultant tissue is formed then used to reconstruct the $A C L$ as per the surgeon's favoured technique. It is common for the tendons to be folded over each other in order to increase the thickness of the donor graft [6].

A systematic review and meta-analysis of prospective studies with a minimum 5-year follow-up found that graft rupture rates varied from $1.8 \%$ to $10.4 \%$, with a pooled percentage of $5.8 \%$ (all grafts were autografts) [7]. In addition to the rate of graft rupture, it is also relevant to know whether predictors (ie, patient and surgical variables) can be used to identify at risk individuals. Risk factors for $\mathrm{ACL}$ graft rupture have been studied with increasing frequency and patient variables that have been associated with graft rupture [8]. Surgical factors that have been indicated include a vertical graft position [9] small graft [10] lax graft $[9,11]$ and hamstring graft [12]. While it is encouraging that so many potential factors have been identified, since all of these variables other than graft diameter are categorical to further investigate the relationship between graft diameter sizes and rupture rate. From this study, it can be seen that the smallest graft size is over represented, with $20 \%$ of patients sustaining graft ruptures [13]. Graft diameter has received recent attention after the publication by Magnussen, et al. which showed that small hamstring grafts were a predictor of early graft failure [14]. Park and colleagues also concluded greater graft rupture rates in patients with a graft size of less than $8 \mathrm{~mm}$ in a mostly nonathletic population at an average 4.5-year follow-up [10]. The frequency distribution between graft size and rupture rates, however, showed that the smallest graft size $(7 \mathrm{~mm})$ was over represented for graft failure (20\% ruptures).

According to Conte, et al. [5] grafts less than $8 \mathrm{~mm}$ in diameter are a risk factor for poor patient outcomes, with an increase in failure rates, especially in patients younger than 20 years [15]. The option adopted in the present technique was to make quadrupled each one of hamstring tendons to get 8 strands ACL graft, in order to increase graft diameter size which gives higher knee stability and long-term survival of the graft.

\section{Conclusion}

The aim of using this technique is increasing graft diameter size by increasing number of folds regardless fixation methods, to ensure long-term survival of small-sized hamstring tendon graft tissue which is common in our population especially non-athletic.

\section{References}

1. Saltzman BM, Cvetanovich GL, Nwachukwu BU, et al. (2016) Economic analyses in anterior cruciate ligament reconstruction: A qualitative and systematic review. Am J Sports Med 44: 1329-1335.

2. Duchman KR, Lynch TS, Spindler KP (2017) Graft selection in anterior cruciate ligament surgery: Who gets what and why? Clin Sports Med 36: 25-33.

3. Struewer J, Ziring E, Oberkircher L, et al. (2013) Isolated anterior cruciate ligament reconstruction in patients aged fifty years: Comparison of hamstring graft versus bone- patellar tendon-bone graft. Int Orthop 37: 809-817.

4. Ahn JH, Lee SH (2007) Anterior cruciate ligament double-bundle reconstruction with hamstring tendon autografts. Arthroscopy 23: 101-109.

5. Conte EJ, Hyatt AE, Gatt CJ Jr, et al. (2014) Hamstring autograft size can be predicted and is a potential risk factor for anterior cruciate ligament reconstruction failure. Arthroscopy 30: 882-890.

6. Koh HS, In Y, Kong CG, et al. (2010) Factors affecting patients' graft choice in anterior cruciate ligament reconstruction. Clin Orthop Surg 2: 69-75.

7. Wright RW, Magnussen RA, Dunn WR, et al. (2011) Ipsilateral graft and contralateral $A C L$ rupture at five years or more following $A C L$ reconstruction: A systematic review. J Bone Joint Surg Am 93: 1159-1165.

8. Kaeding CC, Pedroza AD, Reinke EK, et al. (2015) Risk factors and predictors of subsequent $A C L$ injury in either knee after $A C L$ reconstruction: Prospective analysis of 2488 primary ACL reconstructions from the MOON cohort. Am J Sports Med 43: 1583-1590.

9. Bourke HE, Gordon DJ, Salmon L, et al. (2012) The outcome at 15 years of endoscopic anterior cruciate ligament reconstruction using hamstring tendon autograft for isolated anterior cruciate ligament rupture. J Bone Joint Surg Br 94: 630-637.

10. Park SY, Oh H, Park S, et al. (2013) Factors predicting hamstring tendon autograft diameters and resulting failure rates after anterior cruciate ligament reconstruction. Knee Surg Sports Traumatol Arthrosc 21: 1111-1118.

11. Pinczewski LA, Lyman J, Salmon LJ, et al. (2007) A 10-year comparison of anterior cruciate ligament reconstructions with hamstring tendon and patellar tendon autograft: A controlled prospective trial. Am J Sports Med 35: 564-574.

12. Maletis GB, Inacio MCS, Desmond JL, et al. (2013) Reconstruction of the anterior cruciate ligament: Association of graft choice with increased risk of early revision. Bone Joint J 95B: 623-628.

13. Kate EW, Julian AF, Warren BL, et al. (2014) Younger patients are at increased risk for graft rupture and contralateral injury after anterior cruciate ligament reconstruction. Am J Sports Med. 42: 641-647.

14. Magnussen RA, Lawrence JT, West RL, et al. (2012) Graft size and patient age are predictors of early revision after anterior cruciate ligament reconstruction with hamstring autograft. Arthroscopy 28: 526-531.

15. Iriuchishima T, Shirakura K, Yorifuji H, et al. (2013) Size comparison of $A C L$ footprint and reconstructed auto graft. Knee Surg Sports Traumatol Arthrosc 21: 797-803. 